Received: 13 March 2018

Accepted: 29 August 2018

Published online: 12 September 2018

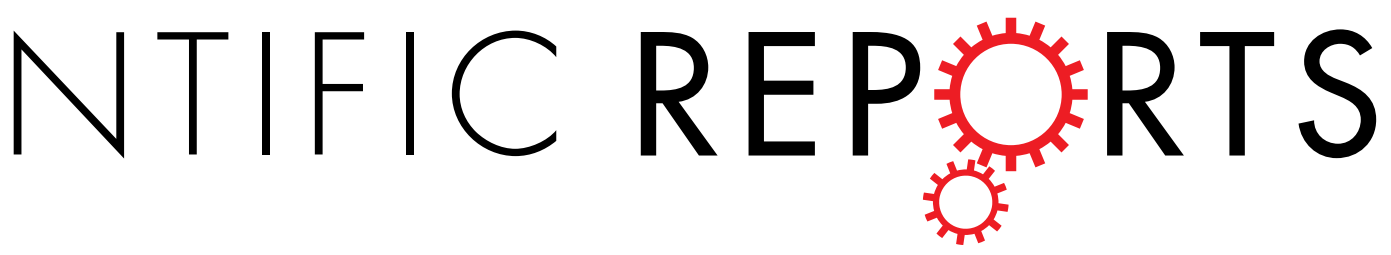

\title{
Critical roles of serotonin-oxytocin interaction during the neonatal period in social behavior in $15 q$ dup mice with autistic traits
}

Masatoshi Nagano ${ }^{1}$, Toru Takumi ${ }^{2,3}$ \& Hidenori Suzuki ${ }^{1,3}$

Disturbance of neurotransmitters and neuromodulators is thought to underlie the pathophysiology of autism spectrum disorder (ASD). Studies of 15q dup mouse models of ASD with human 15q11-13 duplication have revealed that restoring serotonin $(5-\mathrm{HT})$ levels can partially reverse ASD-related symptoms in adults. However, it remains unclear how serotonin contributes to the behavioral symptoms of ASD. In contrast, oxytocin (OXT) has been found to involve social and affiliative behaviors. In this study, we examined whether serotonin-OXT interaction during the early postnatal period plays a critical role in the restoration of social abnormality in $15 q$ dup mice. OXT or the $5-\mathrm{HT}_{1 \mathrm{~A}}$ receptor agonist $80 \mathrm{H}$-DPAT treatment from postnatal day 7 (PD7) to PD21 ameliorated social abnormality in the threechamber social interaction test in adult $15 q$ dup mice. The effect of $80 \mathrm{OH}$-DPAT was inhibited by blockade of OXT receptors in 15q dup mice. Thus, serotonin-OXT interaction via $5-\mathrm{HT}_{1 \mathrm{~A}}$ receptors plays a critical role in the normal development of social behavior in $15 q$ dup mice. Therefore, targeting serotonin-OXT interaction may provide a novel therapeutic strategy for treatment of ASD.

Autism spectrum disorder (ASD) is a neurodevelopmental disorder defined by deficits in social communication and interaction, as well as restricted and repetitive patterns of behavior, interests, or activities (DSM-5). Although various hypotheses have been proposed regarding the pathophysiology of ASD, it has been confirmed that ASD has a strong genetic basis. Copy number variation $(\mathrm{CNV})$ is a significant risk factor for ASD. Using a chromosome engineering technique, we successfully generated a mouse line with duplication of chromosome 7 corresponding to human chromosome $15 \mathrm{q} 11-13^{1}$, which is one of the CNV loci most frequently identified in cytogenetic abnormalities of $\mathrm{ASD}^{2,3}$. Paternal duplication (15q dup, patDp/+ in ref. $\left.{ }^{1}\right)$ mice exhibit several abnormal behaviors frequently observed in ASD, such as impaired social interaction and communication, behavioral inflexibility and anxiety-like behaviors ${ }^{1}$. Therefore, $15 q$ dup mice retain part of the clinical and genetic features of ASD, thus providing an appropriate model for investigating the pathophysiology and therapeutic intervention of ASD. 15q dup mice also exhibit low serotonin levels in the brain during development ${ }^{4}$. Interestingly, these mice show reversibility of a subset of ASD-related symptoms in adults by the restoration of normal serotonin levels during postnatal development ${ }^{5}$. Although these findings suggest that serotonin may have therapeutic potential for discrete ASD symptoms, the detailed mechanisms of serotonergic modulation remain unclear.

Accumulating evidence indicates that oxytocin (OXT) plays a crucial role in social and affiliative behaviors. Deletion of OXT or the OXT receptor gene results in deficits in social behavior in mice ${ }^{6-9}$. Moreover, single-dose or continuous treatment with OXT improves low sociability in ASD model animals ${ }^{10-15}$ and patients with ASD ${ }^{16-18}$. Therefore, OXT is considered a promising therapeutic candidate for ASD. OXT is released in the brain and acts as a hormone or neuromodulator on a variety of neurotransmitter systems including GABA ${ }^{19,20}$, dopamine ${ }^{21}$ and serotonin ${ }^{22-26}$. Among these mechanisms, the interaction between OXT and serotonin is of particular interest, because both molecules are involved in the control of social behavior ${ }^{27,28}$. OXT receptors are located on serotonergic cells ${ }^{18,20}$, while serotonin $(5-\mathrm{HT})$ receptors are located on OXT neurons ${ }^{29}$. Further, OXT-induced 5-HT release in the nucleus accumbens is reported to support social reward ${ }^{23}$. Considering the reversibility of ASD symptoms

${ }^{1}$ Department of Pharmacology, Graduate School of Medicine, Nippon Medical School, 1-1-5 Sendagi, Bunkyo-ku, Tokyo, Japan. ${ }^{2}$ RIKEN Brain Science Institute, Wako, Saitama, 351-0198, Japan. ${ }^{3}$ Core Research for Evolutional Science and Technology (CREST), Japan Science and Technology Agency, Tokyo, Japan. Correspondence and requests for materials should be addressed to H.S. (email: hsuzuki@nms.ac.jp) 
using a selective serotonin reuptake inhibitor (SSRI) in our previous study ${ }^{5}$, the serotonin-OXT interaction may underlie the reversible effect of serotonin restoration on social behavior. Therefore, in the current study, we examined whether the OXT system is involved in the effects of serotonergic intervention in 15 dup mice during the early postnatal period.

\section{Results}

OXT treatment from PD7 to PD21 ameliorates social abnormality in adult 15q dup mice. Because a similar early intervention study found that the SSRI fluoxetine (FLX) was effective ${ }^{5}$, we examined whether early postnatal OXT treatment ameliorated the abnormal social interaction observed in adult $15 q d u p$ mice. To this end, we treated mice with OXT from postnatal day (PD) 7 to PD21 and examined their behavior at $8-10$ weeks of age.

In the open field (OF) test, the OXT treatment did not affect the total distance traveled in the OF in either wild type (WT) or $15 q d u p$ mice (Fig. 1a; two-way ANOVA; interaction: $F_{1,43}=0.238, p=0.628$; drug: $F_{1,43}>0.001, p=0.981$; genotype: $F_{1,43}=0.066, p=0.798$; Dunnett's test: WT-saline, $6,650 \pm 508 \mathrm{~cm}$ vs. WT-OXT, $6,390 \pm 540 \mathrm{~cm}, p=0.974$ : WT-saline vs. $15 q d u p$-saline, $6,530 \pm 351 \mathrm{~cm}, p=0.997$ : WT-saline vs. $15 q d u p$-OXT, $6,770 \pm 488 \mathrm{~cm}, p=0.996$ ). As for the time spent in the center, a significant drug effect was observed. WT and $15 q d u p$ mice who received OXT treatment spent more time in the center than those with the saline treatment, respectively. On the other hand, there were no significant differences between WT mice received saline and $15 q$ $d u p$ mice treated with OXT (Fig. $1 b$; two-way ANOVA; interaction: $F_{1,43}=0.053, p=0.819$; drug: $F_{1,43}=16.76$, $p<0.01$; genotype: $F_{1,43}=5.87, p=0.020$; Dunnett's test: WT-saline, $115 \pm 14.9 \mathrm{~s}$ vs. WT-OXT, $181 \pm 15.2 \mathrm{~s}$, $p=0.017$ : WT-saline vs. $15 q d u p$-saline, $80.9 \pm 12.0 \mathrm{~s}, p=0.301$ : WT-saline vs. $15 q d u p$-OXT, $140 \pm 15.5 \mathrm{~s}$, $p=0.448)$.

In the three-chamber social interaction (3-CSI) test, there was no difference between the time spent in the areas around the cage with the stranger mouse and in the areas around the empty cage in $15 q d u p$ mice (Fig. 1c; stranger cage, $188 \pm 24.0 \mathrm{~s}$ vs. empty cage, $176 \pm 25.0 \mathrm{~s}, \mathrm{t}_{10}=0.272, p=0.791$, Cohen's $d=0.15$ ), as we reported in previous studies ${ }^{1,5}$. In contrast, the $15 q d u p$ mice neonatally treated with OXT spent significantly more time around the cage with the stranger mouse than around the empty cage (stranger cage, $226 \pm 17.1 \mathrm{~s}$ vs. empty cage, $110 \pm 10.9 \mathrm{~s}^{\mathrm{t}} \mathrm{t}_{15}=4.858, p<0.001$, Cohen's $d=2.02$ ), as did the saline-treated WT mice (stranger cage, $246 \pm 10.3 \mathrm{~s}$ vs. empty cage, $145 \pm 12.4 \mathrm{~s}, \mathrm{t}_{10}=5.980, p<0.001$, Cohen's $d=2.65$ ). In contrast, neonatal OXT treatment did not affect the tendency to spend more time around the stranger cage in WT mice (stranger cage, $209 \pm 38.2 \mathrm{~s}$ vs. empty cage, $77.0 \pm 17.0 \mathrm{~s}, \mathrm{t}_{8}=3.165, p=0.013$, Cohen's $d=1.48$ ).

5- $\mathrm{HT}_{1 \mathrm{~A}}$ receptor agonist treatment from PD7 to PD21 ameliorates the social abnormality in adult $15 q$ dup mice. We next examined the mechanisms underlying the restoration of social behavior induced by FLX or OXT in $15 q$ dup mice. Because $5-\mathrm{HT}_{1 \mathrm{~A}}$ receptor agonists reportedly elevate the plasma OXT levels in rodents ${ }^{28,29}$, we focused on $5-\mathrm{HT}_{1 \mathrm{~A}}$ receptor-mediated effects. Therefore, we treated mice with $8 \mathrm{OH}-\mathrm{DPAT}$, a $5-\mathrm{HT}_{1 \mathrm{~A}}$ receptor agonist, from PD7 to PD21, and examined their behavior in adulthood.

In the OF test, the total distance traveled in the OF showed no difference among WT and $15 q$ dup mice treated with $8 \mathrm{OH}-\mathrm{DPAT}$ or saline (Fig. $2 \mathrm{a}$; two-way ANOVA; interaction: $F_{1,44}=0.005, p=0.944$; drug: $F_{1,44}=0.892$, $p=0.350$; genotype: $F_{1,44}=0.457, p=0.503$; Dunnett's test: WT-saline, $5,890 \pm 388 \mathrm{~cm}$ vs. WT-DPAT, $6,280 \pm 233 \mathrm{~cm}, p=0.831$ : WT-saline vs. $15 q d u p$-saline, $6,180 \pm 465 \mathrm{~cm}, p=0.922$ : WT-saline vs. $15 q d u p$-DPAT, $6,520 \pm 415 \mathrm{~cm}, p=0.534)$. As shown in OXT treatment, a significant drug effect was observed for the time spent in the center in $8 \mathrm{OH}-\mathrm{DPAT}$ treatment. On the other hand, there were no significant differences among groups (Fig. 2b; two-way ANOVA; interaction: $F_{1,44}=1.287, p=0.263$; drug: $F_{1,44}=5.339, p=0.026$; genotype: $F_{1,44}=4.888, p=0.032$; Dunnett's test: WT-saline, $93.4 \pm 11.9 \mathrm{~s}$ vs. WT-DPAT, $134 \pm 16.1 \mathrm{~s}, p=0.062$ : WT-saline vs. $15 q d u p$-saline, $80.8 \pm 9.7 \mathrm{~s}, p=0.807$ : WT-saline vs. $15 q d u p$-DPAT, $94.6 \pm 8.3 \mathrm{~s}, p=1.000$ ).

In the 3-CSI test, saline-treated $15 q d u p$ mice showed no difference in the time spent in the areas around the cage with the stranger mouse and the empty cage (Fig. 2c; $15 q d u p$-saline, stranger cage, $200 \pm 17.9$ s vs. empty cage, $161 \pm 21.6 \mathrm{~s}, \mathrm{t}_{11}=1.080, p=0.303$, Cohen's $d=0.56$ ), whereas $8 \mathrm{OH}$-DPAT-treated $15 q d u p$ mice spent more time in the area around the cage with the stranger mouse than in the area around the empty cage (15qdup-DPAT, stranger cage, $268 \pm 17.1 \mathrm{~s}$ vs. empty cage, $130 \pm 13.2 \mathrm{~s}, \mathrm{t}_{13}=4.993, p<0.001$, Cohen's $\left.d=2.40\right)$. Neonatal treatment with $8 \mathrm{OH}$-DPAT did not significantly affect the behavior in WT mice in adulthood (WT-DPAT, stranger cage, $215 \pm 26.6 \mathrm{~s}$ vs. empty cage, $119 \pm 10.5 \mathrm{~s}, \mathrm{t}_{11}=3.257, p=0.008$, Cohen's $d=1.37$ ).

We then examined whether $8 \mathrm{OH}-\mathrm{DPAT}$ affects plasma OXT levels in WT and $15 q d u p$ mice. Ten minutes after subcutaneous injection of $8 \mathrm{OH}$-DPAT at $0.5 \mathrm{mg} / \mathrm{kg}$, the plasma OXT levels were significantly increased in both WT and $15 q d u p$ mice at 3 weeks old (Fig. $3 \mathrm{a}$; WT-saline, $0.895 \pm 0.09 \mathrm{ng} / \mathrm{mL}$ vs. WT-DPAT, $1.17 \pm 0.05 \mathrm{ng} / \mathrm{mL}$, $\mathrm{t}_{8}=2.606, p=0.031$ : Fig. $3 \mathrm{~b} ; 15 q d u p$-saline, $0.824 \pm 0.07 \mathrm{ng} / \mathrm{mL}$ vs. $15 q d u p$-DPAT, $1.08 \pm 0.07 \mathrm{ng} / \mathrm{mL}, \mathrm{t}_{8}=2.730$, $p=0.026)$. Basal plasma OXT levels were comparable between WT and $15 q d u p$ mice.

An OXT receptor antagonist treatment from PD7 to PD21 reverses the effect of 80H-DPAT on social abnormality in adult $15 q$ dup mice. Finally, we examined whether the effects of neonatal $8 \mathrm{OH}-\mathrm{DPAT}$ treatment on abnormal social behavior are mediated through OXT receptors. To this end, we administered L-368,899, an OXT receptor antagonist, simultaneously with 8OH-DPAT to mice from PD7 to PD21.

In the OF test, L-368,899 treatment did not affect the total distance traveled in the OF in either WT or $15 q$ dup mice (Fig. 4a; one-way ANOVA: $F_{3,47}=2.488, p=0.072$; Dunnett's test: WT-saline, $6,600 \pm 329 \mathrm{~cm}$ vs. WT-L368899, 5,510 $\pm 226 \mathrm{~cm}, p=0.065$ : WT-saline vs. WT-DPAT-L368899, $6,520 \pm 255 \mathrm{~cm}, p=0.996$ : WT-saline vs. $15 q$ dup- DPAT-L368899, $6,510 \pm 483 \mathrm{~cm}, p=0.995)$. The time spent in the center was unaffected by L-368,899 in either WT or $15 q$ dup mice, regardless of co-treatment with $8 \mathrm{OH}-\mathrm{DPAT}$ (Fig. $4 \mathrm{~b}$; one-way ANOVA: 
a
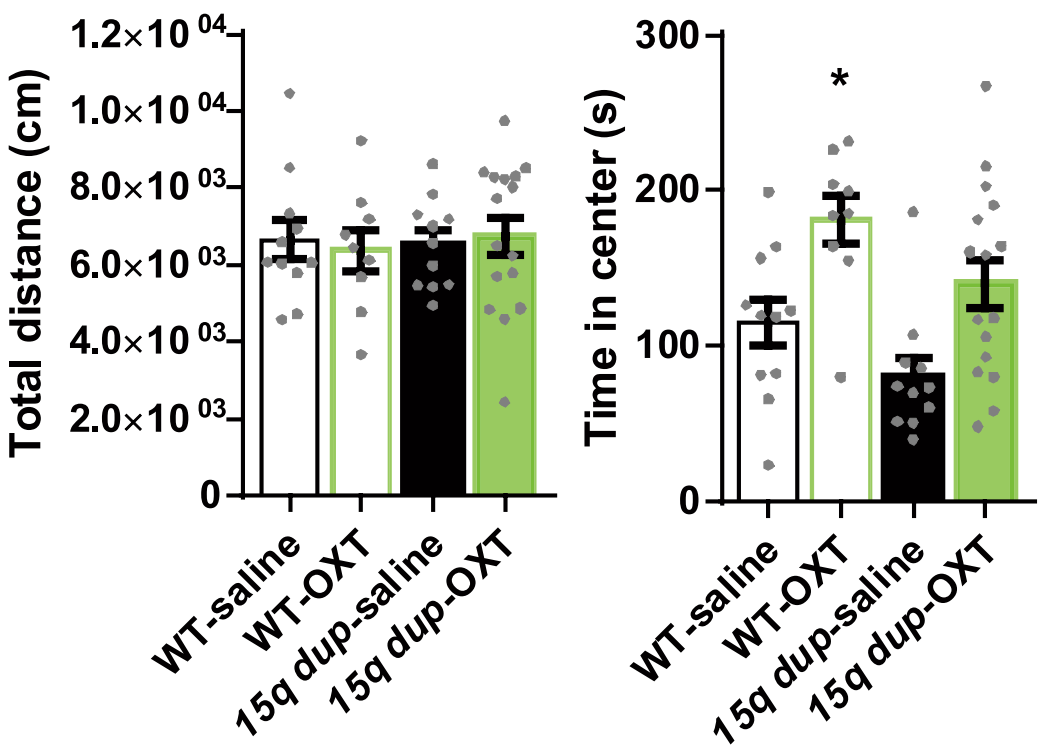

C
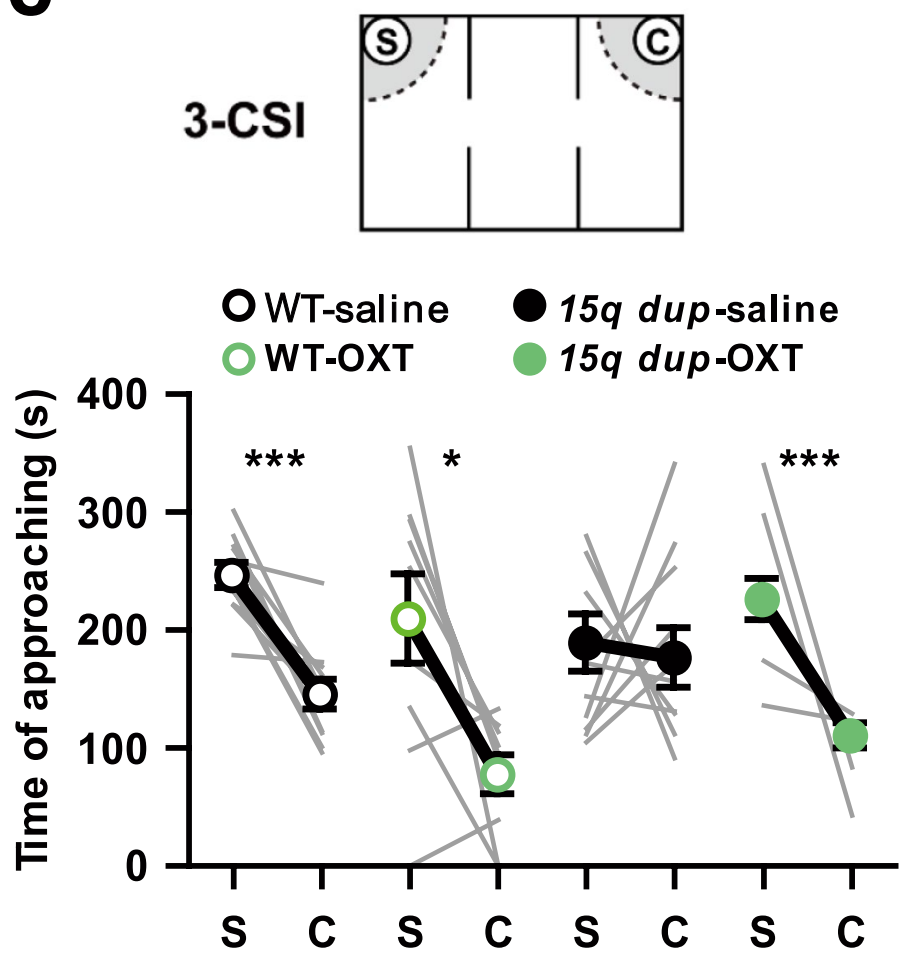

Figure 1. Effects of postnatal OXT treatment on behaviors of adult mice. (a) Total distance in OF test. OXT treatment did not affect the total distance traveled in the OF in either WT or $15 q$ dup mice. (b) Time spent in the center area in OF test. Significant drug effect was observed for the time spent in the center (two-way ANOVA; drug: $\left.F_{1,43}=16.76, p<0.01\right)$. The time spent in the center was significantly increased in WT-OXT mice (Dunnett's test; $p=0.017$ ). (c) Approaching time to the stranger cage (S) and the empty cage $(\mathrm{C})$ in the 3 -CSI test. There was no significant difference between the time spent around the stranger mouse and the empty cage in $15 q d u p$-saline mice (WT-saline: $\mathrm{t}_{10}=5.980, p<0.001$, Cohen's $d=2.65$; WT-OXT: $\mathrm{t}_{8}=3.165, p=0.013$, Cohen's $d=1.48 ; 15 q d u p$-saline: $\mathrm{t}_{10}=0.272, p=0.791$, Cohen's $d=0.15 ; 15 q d u p$-OXT: $\mathrm{t}_{15}=4.858, p<0.001$, Cohen's $d=2.02$; paired t-test). The numbers of mice tested and their litters (mice/litters) in each group were as follows. WT-saline (11/4), WT-OXT (9/4), 15qdup-saline (11/6), 15qdup-OXT (16/5). Data represent the mean \pm SEM. $* p<0.05, * * * p<0.001$. 


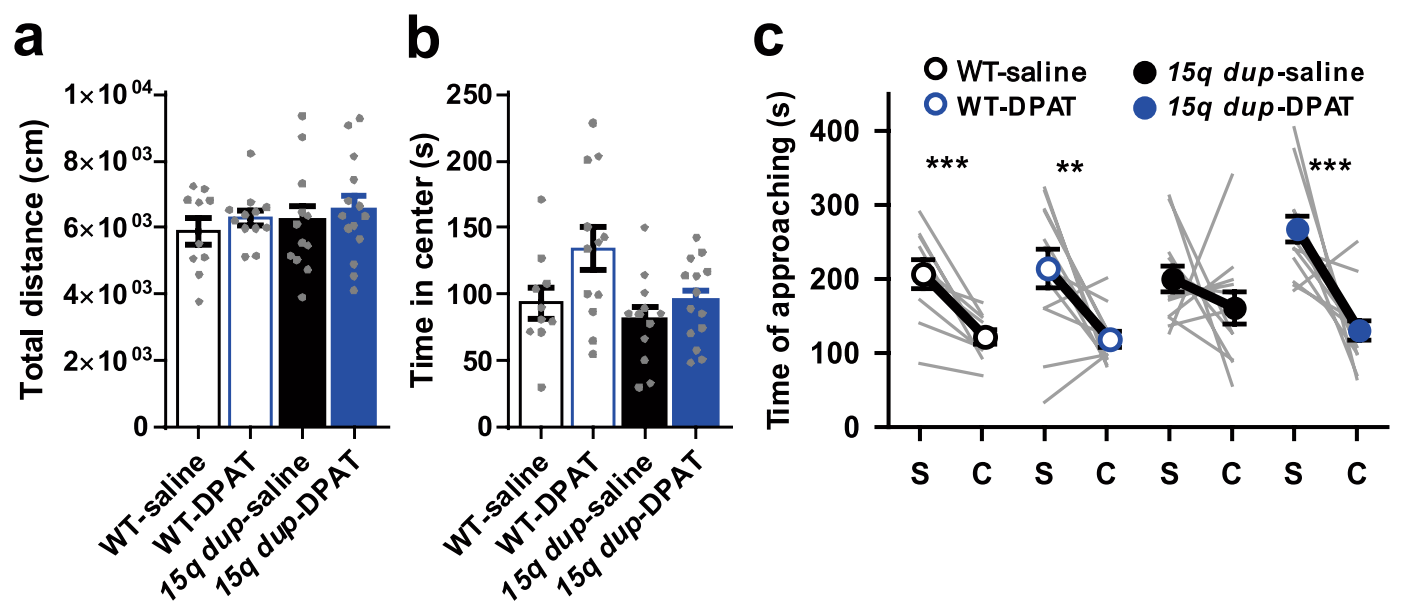

Figure 2. Effects of postnatal 8OH-DPAT treatment on behaviors of adult mice. (a) Total distance in OF test. 8OH-DPAT treatment did not affect the total distance traveled in the OF in either WT or $15 q$ dup mice. (b) Time spent in the center area in OF test. Significant drug effect was observed for the time spent in the center (two-way ANOVA; drug: $F_{1,44}=5.339, p=0.026$ ), while there were no significant differences among groups. (c) Approaching time to the stranger cage $(S)$ and the empty cage $(C)$ in 3-CSI test. There was no significant difference between the time spent around the stranger mouse and the empty cage only in $15 q d u p$-saline mice (WT-saline: $\mathrm{t}_{9}=5.028, p<0.001$, Cohen's $d=1.75$; WT-DPAT: $\mathrm{t}_{11}=3.257, p=0.008$, Cohen's $d=1.37 ; 15 q d u p$ saline: $\mathrm{t}_{11}=1.080, p=0.303$, Cohen's $d=0.56 ; 15 q d u p$-DPAT: $\mathrm{t}_{13}=4.993, p<0.001$, Cohen's $d=2.40$; paired $\mathrm{t}$-test). The numbers of mice tested and their litters (mice/litters) in each group are as follows. WT-saline (10/4), WT-DPAT (12/6), 15q dup-saline (12/6), 15qdup-DPAT (14/6). Data represent the mean \pm SEM. $* * p<0.01$, $* * * p<0.001$.

a

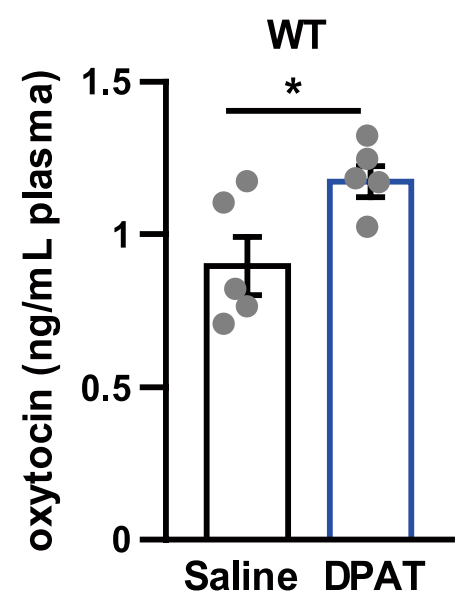

b

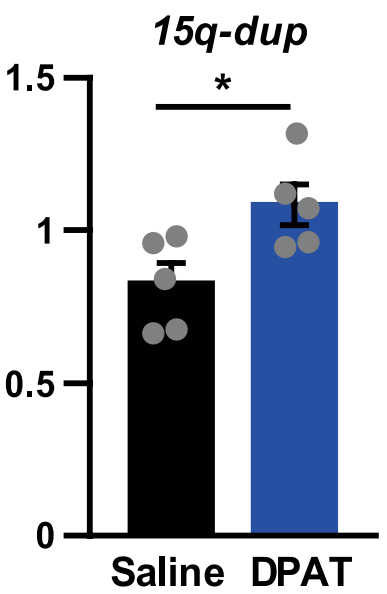

Figure 3. Effects of $8 \mathrm{OH}-\mathrm{DPAT}$ treatment on plasma OXT levels in 3-week-old mice. (a) WT mice (b) $15 q$ dup mice. 8OH-DPAT treatment increased plasma OXT levels both in WT mice (a) WT-saline vs. WT-DPAT, $\left.\mathrm{t}_{8}=2.606, p=0.031\right)$ and $15 q d u p$ mice (b) $15 q d u p$-saline vs. $15 q d u p$-DPAT, $\mathrm{t}_{8}=2.730, p=0.026$; Two-tailed Student's t-tests were applied between treatments in each genotype). The numbers of mice tested and their litters (mice/litters) in each group were as follows. WT-saline (5/3), WT-DPAT (5/3), 15qdup-saline (5/3), 15qdup$\operatorname{DPAT}(5 / 3)$. Data represent the mean \pm SEM. $* p<0.05$.

$F_{3,47}=3.418, p=0.025$; Steel's test: WT-saline, $99.6 \pm 5.8 \mathrm{~s}$ vs. WT-L368899, $142 \pm 15.6 \mathrm{~s}, p=0.25$ : WT-saline vs. WT-DPAT-L368899, $125 \pm 12.4 \mathrm{~s}, p=0.522$ : WT-saline vs. $15 q d u p$-DPAT-L368899, $96.4 \pm 9.3 \mathrm{~s}, p=1$ ).

In the 3-CSI test, L-368,899-treated WT mice spent a similar amount of time around the area of the stranger mouse cage and the empty cage (Fig. 4c; WT-L368899, stranger cage, $154 \pm 19.4 \mathrm{~s}$ vs. empty cage, $134 \pm 15.9 \mathrm{~s}$, $\mathrm{t}_{12}=0.623, p=0.545$, Cohen's $d=0.31$ ). Treatment with L-368,899 reversed the 8OH-DPAT-induced restoration of social behavior in $15 q$ dup mice (Fig. 4c; $15 q$ dup-DPAT-L368899, stranger cage, $179 \pm 18.2 \mathrm{~s}$ vs. empty cage, $135 \pm 13.6 \mathrm{~s}, \mathrm{t}_{11}=4.993, p=0.098$, Cohen's $\left.d=0.80\right)$. On the other hand, WT mice treated with both L-368,899 and $8 \mathrm{OH}-\mathrm{DPAT}$ showed similar behavior to the saline-treated WT mice (Fig. 4c; WT-DPAT-L368899, stranger cage, $206 \pm 14.1 \mathrm{~s}$ vs. empty cage, $121 \pm 16.0 \mathrm{~s}, \mathrm{t}_{13}=2.948, p=0.011$, Cohen's $d=1.50$ ). 


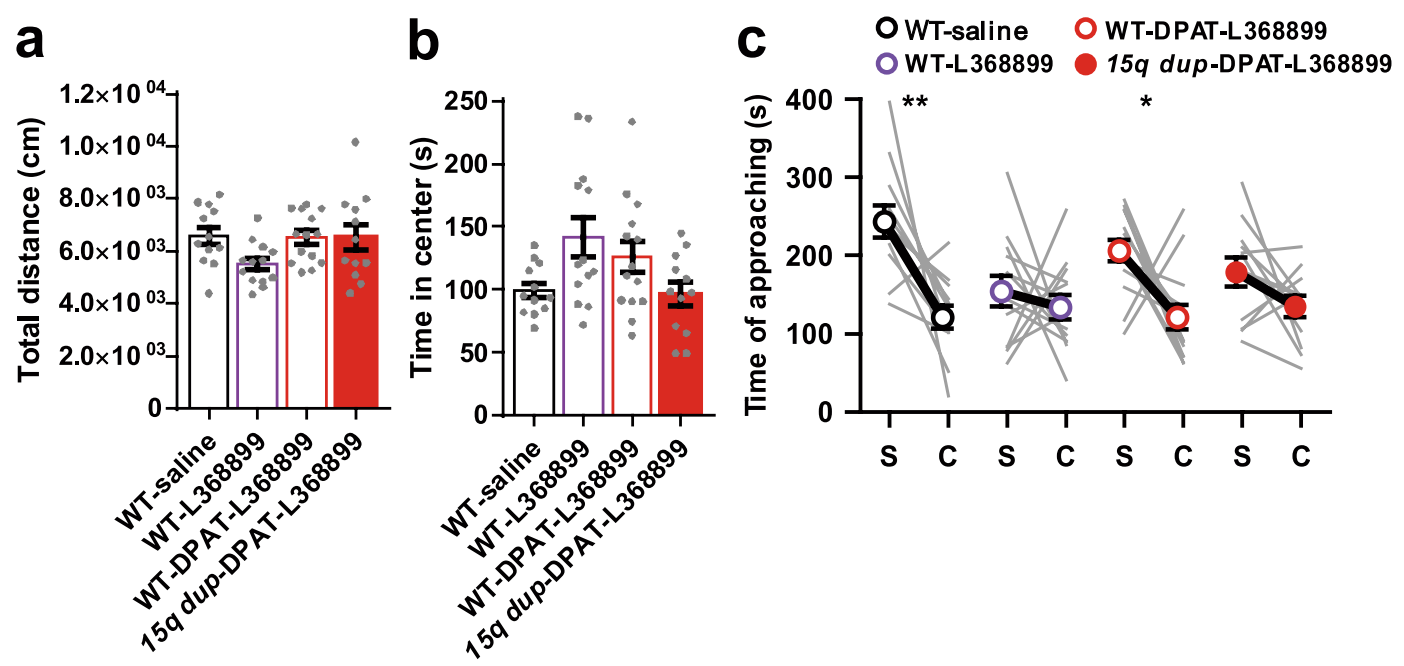

Figure 4. Effects of postnatal L-368,899 treatment on behavior in adult mice. (a) Total distance in OF test. There was no significant difference in the total distance traveled among groups (one-way ANOVA: $\mathrm{F}_{3,47}=2.488$, $p=0.072$; Dunnett's test). (b) Time spent in the center area in the OF test. There was no significant difference in the center time among groups. (one-way ANOVA: $\mathrm{F}_{3,47}=3.418, p=0.025$; Steel's test) (c) Time taken to approach to the stranger cage (S) and the empty cage (C) in 3-CSI test. Under the treatment with L-368,899, WT or $8 \mathrm{OH}$-DPAT-treated $15 q$ dup mice showed no significant difference between the time spent around the stranger mouse and the empty cage (WT-saline: $\mathrm{t}_{11}=3.937, p=0.002$, Cohen's $d=1.96$; WT-L368899: $\mathrm{t}_{12}=0.623, p=0.545$, Cohen's $d=0.31$; WT-DPAT-L368899: $\mathrm{t}_{13}=2.948, p=0.011$, Cohen's $d=1.50 ; 15 q d u p$ DPAT-L368899: $\mathrm{t}_{11}=1.805, p=0.098$, Cohen's $d=0.80$; paired t-test). The numbers of mice tested and their litters (mice/litters) in each group are as follows. WT-saline (12/5), WT-L368899 (13/4), WT-DPAT-L368899 (14/4), $15 q d u p$-DPAT-L368899 (12/4). Data represent the mean \pm SEM. ${ }^{*} p<0.05,{ }^{*} p<0.01$.

\section{Discussion}

The current results revealed that early postnatal OXT treatment ameliorated abnormal social behavior in adult $15 q d u p$ mice with a similar treatment protocol to the method we adopted to successfully restore the abnormal social interaction with FLX in a previous study ${ }^{5}$. Importantly, early postnatal OXT treatment had a long-term benefit on sociability. In addition, treatment with the OXT receptor antagonist L-368,899 from PD7 to PD21 impaired sociability in adulthood in WT mice. Treatment with another OXT receptor antagonist within 24 hours after birth was previously reported to impair sociability in adulthood in female mice ${ }^{30}$. These findings suggest the importance of OXT during the neonatal period for the development of normal social behavior in adulthood. Accordingly, many reports have suggested that impairment of the OXT system affects sociability ${ }^{6-9}$, and OXT is effective for restoration of disturbed sociability in a variety of ASD model animals ${ }^{10-15}$. Clinical studies have also reported that symptoms in patients with ASD are partially improved by OXT applied via nasal spray ${ }^{16-18}$. In the current study, OXT treatment was found to be effective for improving social behavior in $15 q d u p$ mice, as observed in other ASD model animals and patients with ASD. These findings suggest that $15 q d u p$ mice have predictive validity as well as face validity and construct validity as an animal model of ASD.

In the present study, we adopted the subcutaneous injection to deliver precise amount of OXT to immature mice, and observed significant effects of oxytocin on social behaviors compared with the saline. Several studies have shown that intranasal administration of oxytocin increases its concentrations in both brain tissue and plasma $^{31}$ and affects behaviors relating higher brain functions ${ }^{12}$. On the other hand, there also have been several reports showing that peripheral injection of oxytocin increases its concentrations in both brain tissue and plasma with a pharmacokinetic profile different from nasal delivery ${ }^{31}$, and induces some behavioral changes relating higher brain functions ${ }^{14}$, consistent with the present results. Further, because we administered a pharmacological dose of oxytocin (i.e. an excess dose compared with endogenous peptide dose) to postnatal mice, which may have more immature brain-blood barrier than adult mice, oxytocin could more easily penetrate the brain-blood barrier.

The current findings demonstrated that the $5-\mathrm{HT}_{1 \mathrm{~A}}$ receptor agonist $8 \mathrm{OH}$-DPAT administered in the early postnatal period ameliorated the impaired social behavior in adult $15 q$ dup mice, similar to the effects of treatment with FLX. These results suggest that $5-\mathrm{HT}_{1 \mathrm{~A}}$ receptors may be at least partially involved in the restorative effects of early serotonergic intervention on abnormal sociability in adult $15 q d u p$ mice. $5-\mathrm{HT}_{1 \mathrm{~A}}$ receptor agonists have been reported to elevate the plasma OXT levels in rodents ${ }^{32,33}$. OXT neurons are located in the hypothalamus and express 5- $\mathrm{HT}_{1 \mathrm{~A}}$ receptors ${ }^{29}$. In the present study, we confirmed that $8 \mathrm{OH}$-DPAT increased plasma OXT levels both in WT and 15q dup mice. Further, the OXT receptor antagonist L-368,899 blocked the 8OH-DPAT-induced restoration of social behavior in $15 q d u p$ mice. These lines of evidence suggest that the OXT system acts downstream of the serotonergic systems to facilitate normal development of social behavior. However, we found that L-368,899 blocked normal social behavior in WT mice, but not in WT mice treated with 8OH-DPAT. The reason for the discrepancy in these effects is unclear. Experimental conditions such as dose or specificity of the 
antagonist may have affected the results. Alternatively, because OXT receptors are expressed on serotonergic cells $^{22,24}$, the OXT system may inversely exert effects on serotonergic neurons. Further, there may be some differences in responsiveness to OXT between WT and $15 q d u p$ mice, including the OXT receptor expression level in the brain. Interestingly, it has been previously reported that functional serotonin-OXT interactions are altered in the brain in patients with $\mathrm{ASD}^{34}$.

In conclusion, the current findings indicate that OXT is effective for tr eating impaired sociability in $15 q d u p$ mice, consistent with the effects observed in other ASD model animals with different genetic mutations ${ }^{10,13-15}$. In addition to the OXT system, serotonin-OXT interactions via $5-\mathrm{HT}_{1 \mathrm{~A}}$ receptors play a critical role in the normal development of social behavior. Therefore, targeting serotonin-OXT interactions may provide another novel therapeutic strategy for treatment of ASD.

\section{Methods}

Animals. We tested male $15 q d u p$ mice ${ }^{1,5}$ and their littermate male wild type (WT) C57BL/6J mice. All mice were kept under constant temperature $\left(22 \pm 1^{\circ} \mathrm{C}\right)$ on a regular light/dark cycle (lights on from 06:00 to 20:00 h), with free access to food and water. All experiments were conducted in accordance with the National Institutes of Health Guide for the Care and Use of Laboratory Animals. This study was reviewed by the Institutional Animal Care and Use Committee and approved by the President of the Nippon Medical School (Approval Number: 27-035).

Drug treatment. (R)-(+)-8-hydroxy-2-(di- $n$-propylamino)tetralin hydrobromide (8OH-DPAT; Sigma, USA), a specific $5-\mathrm{HT}_{1 \mathrm{~A}}$ receptor full agonist, L-368,899 hydrochloride (Tocris, UK), an OXT receptor antagonist, or OXT (Sigma, USA) was dissolved in saline and subcutaneously injected to pups (8OH-DPAT, $0.5 \mathrm{mg} / \mathrm{kg}$; L-368,899, $3 \mathrm{mg} / \mathrm{kg}$; OXT 83 I.U. [0.2-0.26 mg]/kg) every day from PD7 to PD21.

The treatment doses of $8 \mathrm{OH}-\mathrm{DPAT}{ }^{35}, \mathrm{~L}-368,899^{36,37}$ and $\mathrm{OXT}^{30}$ were determined based on previous reports. The treatment duration of each drug was determined based on our previous reports ${ }^{5}$. After weaning at PD21, the mice were divided into groups of two to five mice per cage. Saline was used as a control.

Measurement of plasma OXT. Three-week-old $15 q$ dup and wild type (WT) mice were subcutaneously administered with saline or $8 \mathrm{OH}$-DPAT $(0.5 \mathrm{mg} / \mathrm{kg})$. Ten minutes later, the mice were decapitated under deep anesthesia with halothane and the blood was collected. The plasma OXT was assayed with an extraction-free enzyme immunoassay kit (Phoenix Pharmaceuticals, Inc., USA).

Behavioral tests. All behavioral tests were performed between 08:30 and 14:00 h on mice at 8-10 weeks of age ( $n=9-16$ per group). The apparatuses and software used for behavioral analyses were produced by O'Hara \& Co. Ltd. (Tokyo, Japan). The OF and 3-CSI tests were conducted as previously described ${ }^{5}$. Briefly, the OF test was conducted prior to the 3-CSI test. Behavior was monitored for $15 \mathrm{~min}$ and recorded with a CCD camera connected to a personal computer. The ambulation distance and the time spent in the center of the field (a central square of $30 \times 30 \mathrm{~cm}$ ) were automatically recorded and analyzed. For the 3-CSI test, each subject mouse was acclimated to the test box by free exploration for $5 \mathrm{~min}$ before the test. Subsequently, an unfamiliar younger WT C57BL/6J male mouse (stranger mouse) that had no prior contact with the subject mouse was placed in a wire cage at one corner, while the other cage at the opposite corner remained empty. After the subject mouse was placed in the middle chamber to freely move throughout all three chambers, the behavior was recorded for $10 \mathrm{~min}$ and the time spent in the interaction zone (an area within $9 \mathrm{~cm}$ from the edge of the wire cage) was automatically measured for the approaching time. Because the 3-CSI system used in the present study can automatically calculate the duration of stay time (in each cage) as the approaching time without distinguishing the posture of the mice (facing to the stranger mouse or not), we manually examined the posture of the mice around the stranger cage by placing a video camera near the cage in seven mice and compared the result of the automated system in a preliminary experiment. We found that the time spent interaction zone obtained from the automated system was highly correlated with the time of facing to the stranger mouse manually counted $(r=0.93)$, confirming examined approaching (stay) time around the stranger cage as a reliable index of social behavior in the system ${ }^{1,5}$. The observer was blinded to the mouse genotypes until all behavioral tests were finished.

Statistics. All data are expressed as means \pm SEM. For OF tests, two-way ANOVA (Figs 1a,b and 2a,b) or one-way ANOVA (Fig. 4a,b) was used. Then, as post hoc analysis, Dunnett's test (Figs 1a,b, 2a,b and 4a) or Steel's test (Fig. 4b) was used for comparison of values between individual groups. For 3-CSI tests, a paired t-test was used to compare the time spent in the interaction zone of the cage with the stranger mouse with that of the empty cage within each strain or within each treatment group, following papers ${ }^{38,39}$ by Crawley's group. Cohen's $d$ values were calculated online (http://www.socscistatistics.com/effectsize/Default3.aspx) to express the effect size for the significant pairwise comparisons (Cohen's $d$ values greater than 0.5 were considered to be medium in strength, while values greater than 0.8 were considered to have a large effect size $)^{34}$. The analyses were performed using GraphPad Prism software (San Diego, USA) and KyPlot software (KyensLab Inc., Tokyo, Japan). For plasma OXT levels, treatment difference was assessed using two-tailed Student's t-tests in each genotype. $P$-values $<0.05$ were considered statistically significant.

\section{Data Availability}

The datasets generated during and/or analyzed during the current study are available from the corresponding author on reasonable request. 


\section{References}

1. Nakatani, J. et al. Abnormal behavior in a chromosome- engineered mouse model for human 15q11-13 duplication seen in autism. Cell 137, 1235-46 (2009).

2. State, M. \& Levitt, P. The conundrums of understanding genetic risks for autism spectrum disorders. Nat Neurosci 14, 1499-06 (2011).

3. Takumi, T. \& Tamada, K. CNV biology in neurodevelopmental disorders. Curr Opin Neurobiol 48, 183-192 (2018).

4. Tamada, K. et al. Decreased exploratory activity in a mouse model of $15 \mathrm{q}$ duplication syndrome; implications for disturbance of serotonin signaling. PLoS ONE 5, e15126, https://doi.org/10.1371/journal.pone.0015126 (2010).

5. Nakai, N. et al. Serotonin rebalances cortical tuning and behavior linked to autism symptoms in 15q11-13 CNV mice. Sci Adv 3, e1603001, https://doi.org/10.1126/sciadv.1603001 (2017).

6. Winslow, J. T. \& Insel, T. R. The social deficits of the oxytocin knockout mouse. Neuropeptides 36, 221-9 (2009).

7. Takayanagi, Y. et al. Pervasive social deficits, but normal parturition, in oxytocin receptor-deficient mice. Proc Natl Acad Sci USA 102, 16096-101 (2005).

8. Pobbe, R. L. et al. Oxytocin receptor knockout mice display deficits in the expression of autism-related behaviors. Horm Behav 61, 436-44 (2012).

9. Sala, M. et al. Mice heterozygous for the oxytocin receptor gene (Oxtr $+/-)$ show impaired social behaviour but not increased aggression or cognitive inflexibility: evidence of a selective haploinsufficiency gene effect. J Neuroendocrinol 25, 107-18 (2013).

10. Sala, M. et al. Pharmacologic rescue of impaired cognitive flexibility, social deficits, increased aggression, and seizure susceptibility in oxytocin receptor null mice: a neurobehavioral model of autism. Biol Psychiatry 69, 875-882 (2011).

11. Teng, B. L. et al. Reversal of social deficits by subchronic oxytocin in two autism mouse models. Neuropharmacology 105, 61-71 (2016).

12. Hara, Y. et al. Oxytocin attenuates deficits in social interaction but not recognition memory in a prenatal valproic acid-induced mouse model of autism. Horm Behav 96, 130-6 (2017).

13. Harony-Nicolas, H. et al. Oxytocin improves behavioral and electrophysiological deficits in a novel Shank3-deficient rat. eLife e18904, https://doi.org/10.7554/eLife.18904 (2017).

14. Peñagarikano, O. et al. Exogenous and evoked oxytocin restores social behavior in the Cntnap2 mouse model of autism. Sci Transl Med 7:271ra8, https://doi.org/10.1126/scitranslmed.3010257 (2015).

15. Meziane, H. et al. An early postnatal oxytocin treatment prevents social and learning deficits in adult mice deficient for Magel2, a gene involved in Prader-Willi syndrome and autism. Biol Psychiatry 78, 85-94 (2015).

16. Watanabe, T. et al. Clinical and neural effects of six-week administration of oxytocin on core symptoms of autism. Brain 138, 3400-12 (2015)

17. Yatawara, C. J., Einfeld, S. L., Hickie, I. B., Davenport, T. A. \& Guastella, A. J. The effect of oxytocin nasal spray on social interaction deficits observed in young children with autism: a randomized clinical crossover trial. Mol Psychiatry 21, 1225-31 (2016).

18. Parker, K. J. et al. Intranasal oxytocin treatment for social deficits and biomarkers of response in children with autism. Proc Natl Acad Sci USA 114, 8119-24 (2017).

19. Knobloch, H. S. et al. Evoked axonal oxytocin release in the central amygdala attenuates fear response. Neuron 73, 553-66 (2012).

20. Marlin, B. J., Mitre, M., D’Amour, J. A., Chao, M. V. \& Froemke, R. C. Oxytocin enables maternal behaviour by balancing cortical inhibition. Nature 520, 499-504 (2015).

21. Young, L. J. \& Wang, Z. The neurobiology of pair bonding. Nat Neurosci 7, 1048-54 (2004).

22. Yoshida, M. et al. Evidence that oxytocin exerts anxiolytic effects via oxytocin receptor expressed in serotonergic neurons in mice. $J$ Neurosci 29, 2259-71 (2009).

23. Dölen, G., Darvishzadeh, A., Huang, K. W. \& Malenka, R. C. Social reward requires coordinated activity of nucleus accumbens oxytocin and serotonin. Nature 501, 179-84 (2013).

24. Pagani, J. H. et al. Raphe serotonin neuron-specific oxytocin receptor knockout reduces aggression without affecting anxiety-like behavior in male mice only. Genes, Brain and Behavior 14, 167-76 (2015).

25. Lefevre, A. et al. Oxytocin and Serotonin Brain Mechanisms in the Nonhuman Primate. J Neurosci 37, 6741-50 (2017).

26. Arakawa, H. Involvement of serotonin and oxytocin in neural mechanism regulating amicable social signal in male mice: Implication for impaired recognition of amicable cues in BALB/c strain. Behav Neurosci 131, 176-91 (2017).

27. Harmer, C. J. et al. Acute SSRI administration affects the processing of social cues in healthy volunteers. Neuropsychopharmacology 28, 148-52 (2003).

28. Crockett, M. J. \& Fehr, E. Social brains on drugs: tools for neuromodulation in social neuroscience. Soc Cogn Affect Neurosci 9, 250-4 (2014).

29. Hunt, G. E., McGregor, I. S., Cornish, J. L. \& Callaghan, P. D. MDMA-induced c-Fos expression in oxytocin-containing neurons is blocked by pretreatment with the 5-HT-1A receptor antagonist WAY 100635. Brain Res Bull 86, 65-73 (2011).

30. Mogi, K., Ooyama, R., Nagasawa, M. \& Kikusui, T. Effects of neonatal oxytocin manipulation on development of social behaviors in mice. Physiology \& Behavior 133, 68-75 (2014).

31. Neumann, I. D., Maloumby, R., Beiderbeck, D. I., Lukas, M. \& Landgraf, R. Increased brain and plasma oxytocin after nasal and peripheral administration in rats and mice. Psychoneuroendocrinology 38, 1985-93 (2013).

32. Bagdy, G. \& Kalogeras, K. T. Stimulation of 5- $\mathrm{HT}_{1 \mathrm{~A}}$ and $5-\mathrm{HT}_{2} / 5-\mathrm{HT}_{1 \mathrm{C}}$ receptors induce oxytocin release in the male rat. Brain Res 611, 330-2 (1993).

33. Vicentic, A., Li, Q., Battaglia, G. \& Van de Kar, L. D. WAY-100635 inhibits 8-OH-DPAT-stimulated oxytocin, ACTH and corticosterone, but not prolactin secretion. Eur J Pharmacol 346, 261-6 (1998).

34. Lefevre, A. et al. Oxytocin Fails to Recruit Serotonergic Neurotransmission in the Autistic Brain. Cereb Cortex, https://doi. org/10.1093/cercor/bhx272 (2017).

35. Nagano, M., Mingyan, L., Inagaki, H., Kawada, T. \& Suzuki, H. Early intervention with fluoxetine reverses abnormalities in the serotonergic system and behavior of rats exposed prenatally to dexamethasone. Neuropharmacology 63, 292-300 (2012).

36. Pawel, K. et al. Molecular, immunohistochemical, and pharmacological evidence of oxytocin's role as inhibitor of carbohydrate but not fat intake. Endocrinology 151, 4736-44 (2010).

37. Olszewski, P. K., Waas, J. R., Brooks, L. L., Herisson, F. \& Levine, A. S. Oxytocin receptor blockade reduces acquisition but not retrieval of taste aversion and blunts responsiveness of amygdala neurons to an aversive stimulus. Peptides 50, 36-41 (2013).

38. Silverman, J. L. et al. GABA $\mathrm{B}$ receptor agonist R-baclofen reverses social deficits and reduced repetitive behavior in two mouse models of autism. Neuropsychopharmacology 40, 2228-39 (2015).

39. Kazdoba, T. M., Hagerman, R. J., Zolkowska, D., Rogawski, M. A. \& Crawley, J. N. Evaluation of the neuroactive steroid ganaxolone on social and repetitive behaviors in the BTBR mouse model of autism. Psychopharmacology 233, 309-23 (2016).

\section{Acknowledgements}

The authors acknowledge the technical assistance by Yasunori Mikahara of department of pharmacology in Nippon Medical School. We thank Benjamin Knight, MSc, from Edanz Group (www.edanzediting.com/ac) for editing a draft of this manuscript. This work was supported in part by MEXT KAKENHI Grant Numbers 25460345 (to H.S.), 26461554 and 17K10085 (to M.N.), 16H06316, 16H06463, and 16K13110 (to T.T.), and grants from the Japan Science and Technology Agency, Core Research for Evolutional Science and Technology (to T.T. and H.S.) and the Takeda Science Foundation (to T.T.). 


\section{Author Contributions}

M.N., T.T. and H.S. designed the research. M.N. performed the behavioral experiments. M.N., T.T. and H.S. wrote the manuscript.

\section{Additional Information}

Competing Interests: The authors declare no competing interests.

Publisher's note: Springer Nature remains neutral with regard to jurisdictional claims in published maps and institutional affiliations.

(c) (i) Open Access This article is licensed under a Creative Commons Attribution 4.0 International License, which permits use, sharing, adaptation, distribution and reproduction in any medium or format, as long as you give appropriate credit to the original author(s) and the source, provide a link to the Creative Commons license, and indicate if changes were made. The images or other third party material in this article are included in the article's Creative Commons license, unless indicated otherwise in a credit line to the material. If material is not included in the article's Creative Commons license and your intended use is not permitted by statutory regulation or exceeds the permitted use, you will need to obtain permission directly from the copyright holder. To view a copy of this license, visit http://creativecommons.org/licenses/by/4.0/.

(c) The Author(s) 2018 